\title{
Adrenal Gland Ganglioneuroblastoma
}

National Cancer Institute

\section{Source}

National Cancer Institute. Adrenal Gland Ganglioneuroblastoma. NCI Thesaurus. Code C7646.

A gang lioneuroblastoma arising from the adrenal gland. 